\title{
An Intelligent Access Control System Based on Passive Radio-Frequency Identification
}

\author{
Rong-Shue Hsiao, ${ }^{*}$ Tian-Xiang Chen, Chun-Hao Kao, Hsin-Piao Lin, and Ding-Bing Lin ${ }^{1}$ \\ Department of Electronic Engineering, National Taipei University of Technology, \\ No. 1, Sec. 3, Zhongxiao E. Rd., Taipei 10608, Taiwan, R.O.C. \\ ${ }^{1}$ Department of Electronic and Computer Engineering, National Taiwan University of Science and Technology, \\ No. 43, Keelung Rd., Sec. 4, Taipei 10607, Taiwan, R.O.C.
}

(Received August 30, 2016; accepted January 6, 2017)

Keywords: access control system, tailgate, RFID, computer vision, real-time location system

The conventional radio-frequency identification (RFID)-based access control system cannot prevent unauthorized personnel from following authorized personnel into a restricted area. Thus, security and safety of personnel cannot be guaranteed. In this paper, we propose an intelligent access control system to address this problem. The proposed system consists of an ultrahighfrequency (UHF) RFID subsystem and a computer vision subsystem. The UHF RFID subsystem is responsible for the identification and authentication of personnel while the computer vision subsystem captures the images of personnel and accurately calculates their number. By comparing the number of personnel determined by the two subsystems, the access control problem of tailgating can be effectively solved. The proposed system has competitive advantages in terms of high detection accuracy and user convenience of hands-free access control. It can be used in many organizations that have security requirements.

\section{Introduction}

Conventional access control systems use passive radio-frequency identification (RFID) technology to identify the authority of personnel and control their access. However, the conventional access control systems cannot prevent unauthorized personnel from tailgating authorized personnel into a restricted area. ${ }^{(1)}$ Real-time location systems (RTLSs) can identify, locate, and track people in real time within indoor areas. It has been widely used in safety and security applications. ${ }^{(2-4)}$ However, the conventional ultrahigh-frequency (UHF) RFID-based RTLS suffers from multipath fading and interference in the indoor environment. ${ }^{(5-7)}$ Moreover, passive RFID systems are limited in bandwidth. ${ }^{(8)}$ Generally, the performance of identification and location may seriously deteriorate. To improve the identification reliability and location accuracy, reducing the impact of the multipath effect is a critical issue. One of the techniques for solving this problem is to specialize on antenna designs with an extremely narrow radiation pattern to minimize the percentage of unwanted reads. ${ }^{(5)}$ Nogueira et al. ${ }^{(5)}$ used antenna diversity techniques to reduce the side effects of multipath fading. Vongkulbhisal and Zhao ${ }^{(6)}$ used antenna beam scanning to overcome the effect of multipath reflections on the localization accuracy. Goller et al.(7) combined a computer vision (CV) system with the RFID system to overcome the limitations of limited ${ }^{*}$ Corresponding author: e-mail: rshsiao@ntut.edu.tw http://dx.doi.org/10.18494/SAM.2017.1516 
bandwidth and the inherent multipath channel characteristic. The advancement in computer vision systems has improved identification reliability and location accuracy, which has reached a state to provide reliable object/people tracking at a low cost.

In this paper, we propose a hybrid UHF RFID and computer vision system for identifying, locating, and tracking RFID tags in the detection area of a choke point. The RFID subsystem provides personnel authority identification, while the CV subsystem provides personnel detection and counting. The proposed system was used in experiments on the accuracy of unauthorized tailgating. The results showed that it is effective in solving the problem of tailgate access by unauthorized personnel.

The rest of this paper is organized as follows. In Sect. 2, we describe the proposed method of using the RFID subsystem and computer vision subsystem. The experimental setup and results are presented in Sect. 3. Finally, in Sect. 4, we conclude this paper with a short summary and future work.

\section{Methods}

In this section, we describe our proposed framework in the following subsections: system architecture, RFID subsystem deployment and authority identification, computer vision subsystem and people counting, and the procedure of tailgate detection.

\subsection{System architecture}

To fulfill the requirement of secure access control, we propose an RFID and computer-visionbased hybrid system architecture, as shown in Fig. 1. In addition, a detection area is delimited in front of the door. The RFID subsystem comprises a UHF RFID reader with two antennas and several semipassive tags. The antennas are installed on both sides near the ceiling above the detection area. Authorized personnel wear a badge embedded with a semipassive tag. The computer vision subsystem contains a depth-color camera, which is installed in the ceiling above the detection area to retrieve depth information. The detection area is the overlapped area that is covered by both the sensing range of RFID antennas and the depth-color camera. The computer

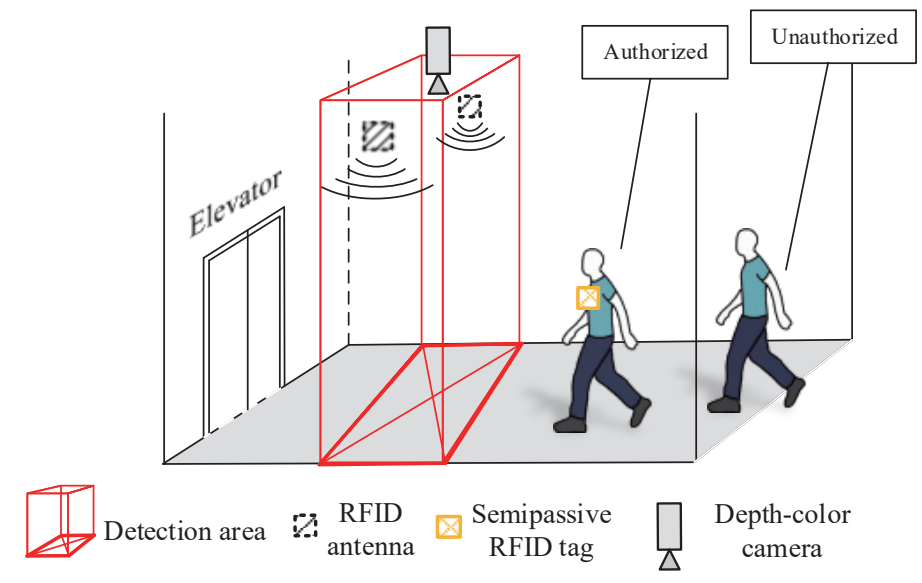

Fig. 1. (Color online) System architecture. 
vision subsystem identifies whether there are persons entering the detection area while the RFID subsystem identifies whether each person is authorized or not.

\subsection{Deployment of RFID subsystem and authority identification}

The RFID reader reads the unique Electronic Product Code (EPC) of a semipassive tag worn by a person in the detection area in order to determine whether that person is authorized or not. The vicinity of the antenna is equipped with two metallic reflectors to limit the direction of radio transceiving, as shown in Fig. 2. In addition, the power of the RFID reader must be set at an adequate level to let each antenna cover half of the area. Then, the transceiving range of these two antennas can cover the rectangular detection area. Thus, either one (or both) of the antennas reads the EPC from the tag. It will be recognized as one person in the detection area. If the power level is too high, the transceiving range of the RFID reader antenna will exceed the detection area, causing another authorized person, who is outside of the detection area to be detected, resulting in a false detection.

\subsection{Computer vision subsystem and people counting}

It is possible for an unauthorized person to tailgate authorized personnel to access the detection area, since the unauthorized personnel cannot be detected by the RFID system. Hence, we use a computer vision system to capture images of personnel in the detection area. Then, the number of people in the detection area can be identified, which includes authorized and unauthorized personnel. The two counting results should be the same if all the personnel are authorized.

Because the transceiving range of RFID antennas is difficult to control, we fit the detection range of a camera to the range of the detection area. Therefore, the detection area is covered by both computer vision and the RFID subsystem.

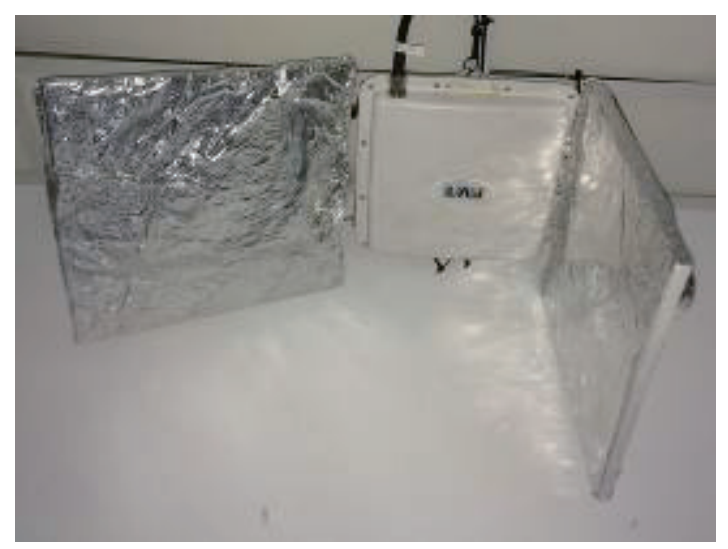

(a)

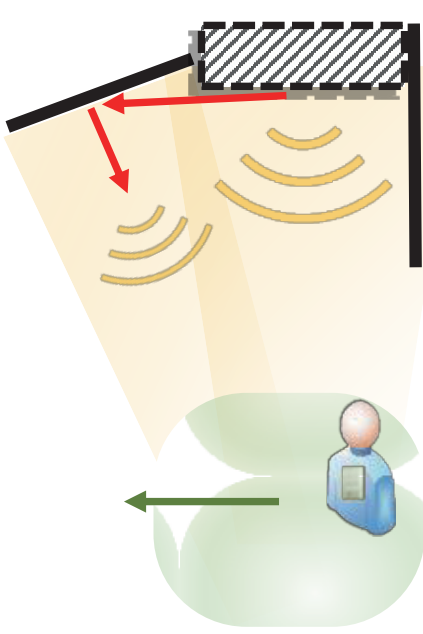

(b)

Fig. 2. (Color online) Installation of antenna: (a) antenna with two metallic reflectors and (b) illustration of the antennas and the authorized person entering the detection area. 
For the computer vision system, the shadow of people may appear in the detection area in advance if an ordinary color camera is used. In the meantime, authorized personnel have not entered the detection area yet, so the RFID subsystem cannot detect any EPC of authorized personnel. The system will yield a false detection. To solve the problem of frequent false detection, we adopt a depth-color camera to estimate the number of people by depth-image-based people counting. ${ }^{(9)}$ As shown in Fig. 3, the yellow points indicate the human body and the green point indicates the position of the human body. It is shown that the person is not in the detection area according to the image of the depth-color camera. Therefore, a person's shadow does not cause false decision.

To accurately count the number of personnel in the detection area, we also define the front and back areas, which are ahead and behind of the detection area, respectively. As shown in Fig. 4, people move in the sequence of front area, detection area, and back area. The CV subsystem captures the image of personnel and decides in which of these three areas the image belongs. In addition, the number of personnel is estimated.

When a person enters the sensing area, the depth-color camera sends contour information to the CV subsystem based on a predefined range. The CV subsystem selects a reasonable size of the image contour for a person. If the contour is too small to be defined as a person in the predefined range, it will be filtered out. The center of gravity $(\mathrm{CoG})$ is calculated for each selected contour of the image to decide in which area the person is located.

\subsection{Detection of unauthorized tailgating}

To detect unauthorized tailgating, the main system needs to make a decision according to the detection result of the RFID subsystem and the CV subsystem, as illustrated in Fig. 5. However, data processing is faster in the CV subsystem than in the RFID subsystem; as a result, the CV subsystem data will arrive faster than the RFID subsystem data. In such a situation, when an authorized person enters the detection area, the main system receives data from the CV subsystem

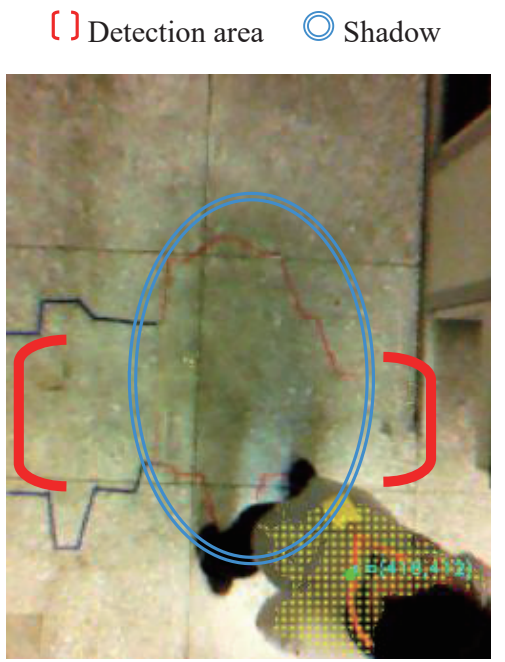

Fig. 3. (Color online) Image of depth-color camera.
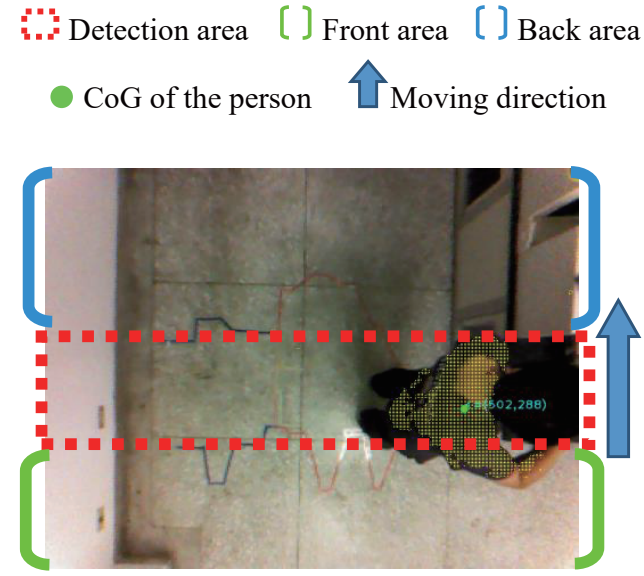

Fig. 4. (Color online) Sensing area definition and location of personnel in the CV subsystem. 


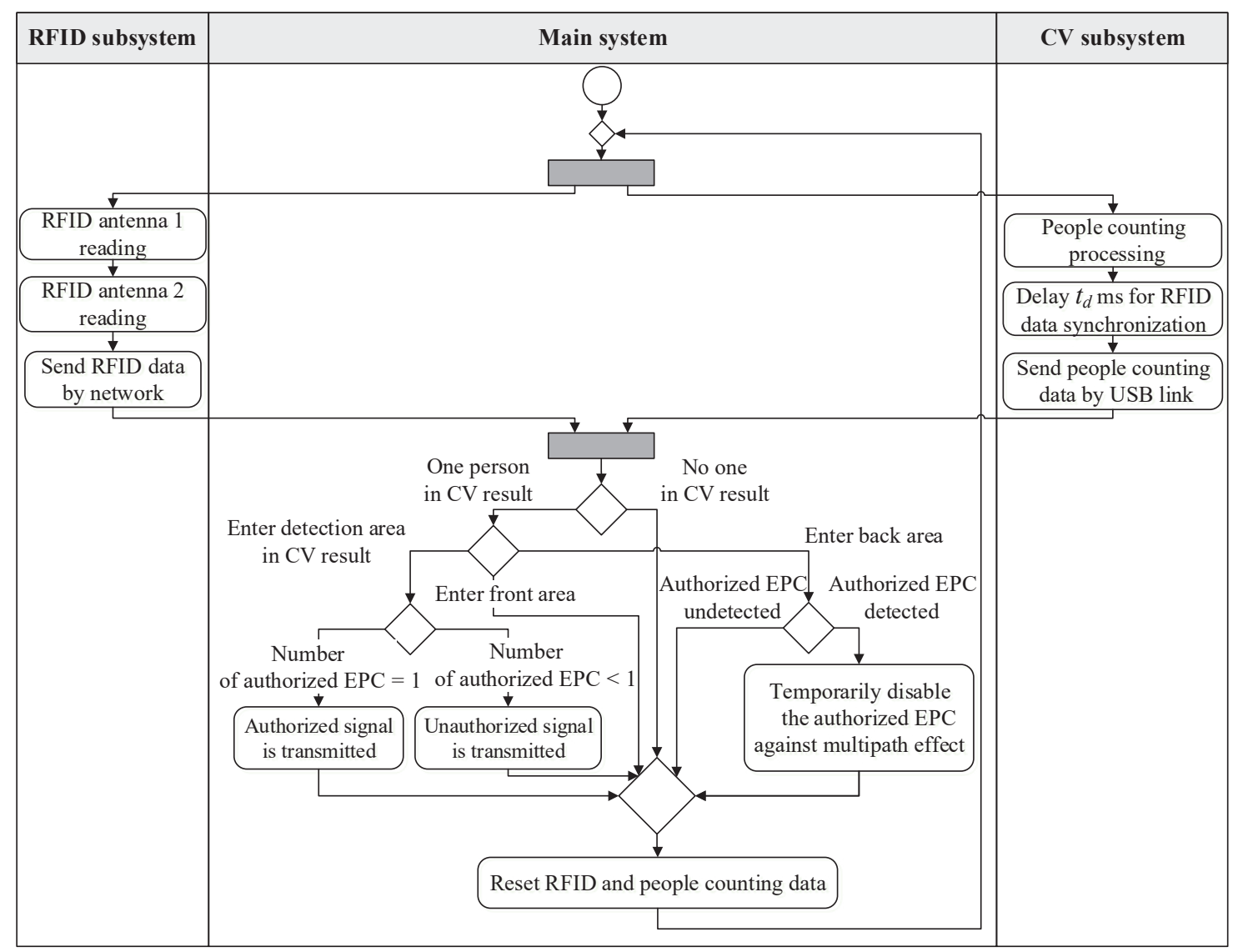

Fig. 5. Activity diagram of tailgate detection.

before it receives the authorized message from the RFID subsystem. Then, the main system decides that this is an unauthorized person, resulting in a false decision. In our system, the operation times of the RFID subsystem and CV subsystem are about 175 and $33 \mathrm{~ms}$, respectively. Therefore, the delay time $\left(t_{d}\right)$ in the CV subsystem must be added before data transmission.

The CV subsystem submits the result to the main system, which indicates one person entering the detection area $(\mathrm{CV}$ result $=1)$. The main system further determines whether it receives authorized data (RFID result $=1$ ) or not (RFID result $=0$ ) from the RFID subsystem. If the RFID result is equal to one, the person is recognized as an authorized person. If not, the person is recognized as an unauthorized person. By calculating the difference between the CV result and RFID result, the main system is capable of detecting a tailgate incident.

Owing to the multipath effect of UHF RFID, the RFID reader continues to read the EPC when an authorized person leaves the detection area and enters the back area. Therefore, when an authorized person enters the back area and an unauthorized person enters the detection area, the RFID subsystem continues to submit authorized data (RFID result $=1$ ), while the CV subsystem also submits a true value $(\mathrm{CV}$ result $=1)$ to the main system, which indicates that the unauthorized person entering the detection area is authorized. To prevent such a false decision, the system temporarily disables an authorized EPC when the authorized person enters the back area. This mechanism effectively offsets the multipath interference property of the UHF RFID and improves the accuracy of tailgate detection. 


\section{Experimental Setup and Results}

As shown in Fig. 6(a), the detection area is set in front of a metal door in order to increase the influence of the multipath effect. Two RFID omnidirectional antennas with metallic reflectors are set on both sides above the detection area. A depth-color camera is deployed above the detection area. A semipassive UHF RFID badge tag is worn by personnel on the chest for identification, as shown in Fig. 6(b).

The passageway in front of the door is restricted to one person passing at a time. A narrow detection area $(185 \mathrm{~cm}$ in width and $50 \mathrm{~cm}$ in length) is delimited in front of the door so that only one person can enter the detection area at a time, as shown in Fig. 7.

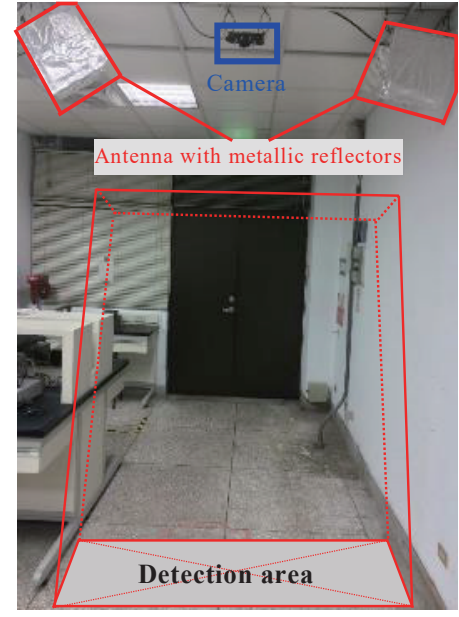

(a)

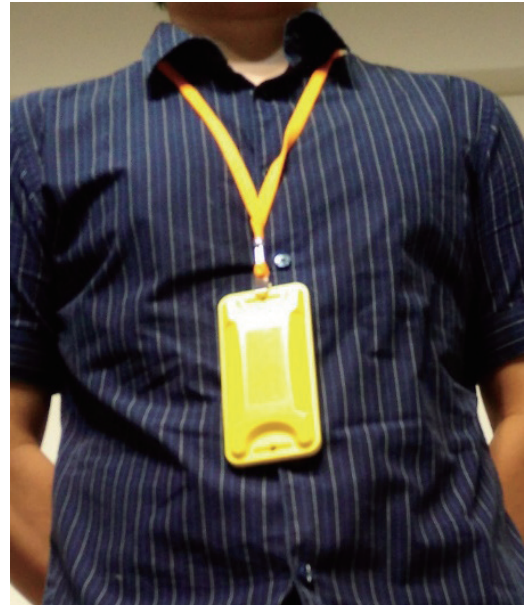

(b)

Fig. 6. (Color online) Experimental setup: (a) experimental environment and (b) the semipassive UHF RFID badge tag.

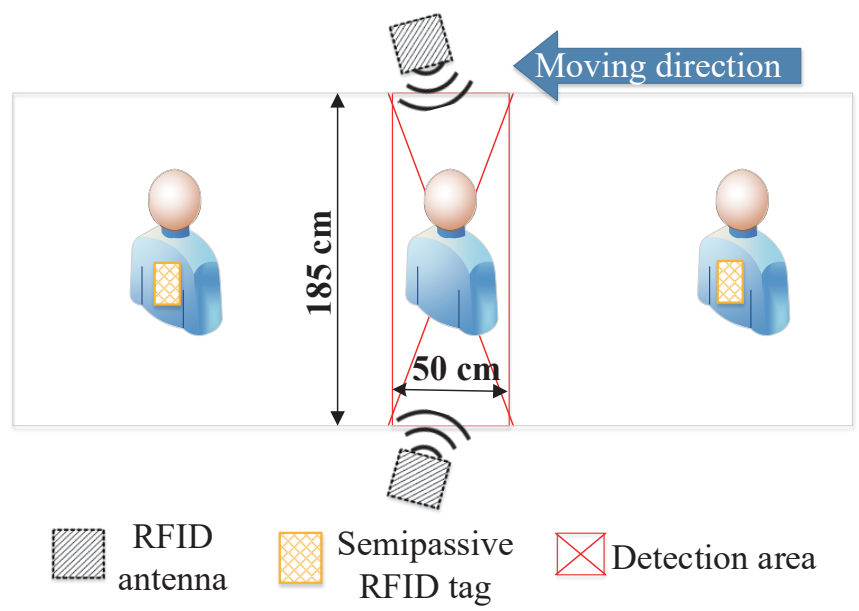

Fig. 7. (Color online) Size of detection area. 
Table 1

Experimental result of authority detection.

\begin{tabular}{lcc}
\hline & \multicolumn{2}{c}{ System judgment } \\
\cline { 2 - 3 } & Positive & Negative \\
\hline Reality: unauthorized & $100 \%$ & $0 \%$ \\
Reality: authorized & $0 \%$ & $100 \%$ \\
\hline
\end{tabular}

Table 2

Experimental result of tailgate detection.

\begin{tabular}{lcccccc}
\hline & \multicolumn{5}{c}{ System judgment } \\
\cline { 2 - 7 } & \multicolumn{2}{c}{$80 \mathrm{~cm}$} & \multicolumn{2}{c}{$70 \mathrm{~cm}$} & \multicolumn{2}{c}{$60 \mathrm{~cm}$} \\
\cline { 2 - 7 } & Positive & Negative & Positive & Negative & Positive & Negative \\
\hline Reality: unauthorized tailgate & $100 \%$ & $0 \%$ & $92 \%$ & $8 \%$ & $96 \%$ & $4 \%$ \\
Reality: authorized tailgate & $9 \%$ & $91 \%$ & $17 \%$ & $83 \%$ & $22 \%$ & $78 \%$ \\
\hline
\end{tabular}

Two experiments were conducted: authority detection and unauthorized tailgate. Firstly, we test whether the personnel in the detection area is unauthorized. The system returns a positive value while an unauthorized person is detected. Otherwise, a negative value is returned. As shown in Table 1, the result shows that the system can accurately detect authority in a single-person situation.

In the second experiment, we test the performance of tailgate detection with different distances between moving personnel. If an unauthorized personnel tailgates another authorized personnel, the system returns a positive value. Otherwise, a negative value is returned if the tailgater is also authorized. As shown in Table 2, all the success rates are above $92 \%$ for unauthourized tailgating. In the authourized personnel tailgate situation, the success rates are 91,83 , and $78 \%$ for the intervals of 80,70 , and $60 \mathrm{~cm}$, respectively. When the distance between two persons becomes shorter, the accuracy of system judgement becomes lower. This indicates that the tailgater's RFID signal may be shielded by the preceding personnel and the false rate is increased.

\section{Conclusions}

In this paper, the design of secure access control was presented. To solve the access control problem of unauthorized tailgating, we proposed a decision approach that combines the detection information of RFID and computer vision subsystems. In the proposed RFID subsystem, we used two metallic reflectors to restrict the transceiving range of an RFID reader antenna to reduce the multipath effect. In the proposed computer vision subsystem, we used the depth-color camera to acquire the depth information of an image to count the actual number of personnel. Experimental results demonstrated that the proposed system is effective in solving the unauthorized tailgate problem. The future work will concentrate on reducing the false positive rate. In addition, we will consider the context of multiple persons passing through the passageway and tailgating. The experiment will be conducted in a more general environment as well.

\section{Acknowledgements}

This work was supported in part by the Ministry of Science and Technology under Grant No. MOST 105-2221-E-027-009. 


\section{References}

1 A. Alarcon-Ramirez, M. Martinez-Pabón, and C. Kim: Int. J. Embedded Syst. Appl. 1 (2011) 1.

2 A. Malik: RTLS For Dummies (Wiley, Hoboken, 2009).

3 M. N. Kamel Boulos: Int. J. Health Geogr. 11 (2012) 1.

4 M. F. Abuhan, A. R. Mohamed Shariff, A. Ghiyamat, and A. R. Mahmud: Science Postprint 1 (2013) 1.

5 E. B. Nogueira, M. H. C. Dias, M. Huchard, F. Nadagijimana, and T. P. Vuong: 2013 SBMO/IEEE MTT-S Int. Microwave \& Optoelectronics Conf. (SBMO/IEEE MTT-S, Rio de Janeiro, 2013) pp. 1-5.

6 J. Vongkulbhisal and Y. Zhao: J. Ambient Intell. Smart Environ. 5 (2013) 251.

7 M. Goller, C. Feichtenhofer, and A. Pinz: Proc. 2014 IEEE Int. Conf. RFID (IEEE, Orlando, 2014) pp. 89-96.

8 G. Li, D. Arnitz, R. Ebelt, U. Muhlmann, K. Witrisal, and M. Vossiek: Proc. IEEE Int. Conf. RFID (IEEE, Orlando, 2011) pp. 19-25.

9 J. Liu, Y. Liu, G. Zhang, P. Zhu, and Y. Q. Chen: Pattern Recognit. Lett. 53 (2015) 16. 